\title{
Semi Bounded Solution of Hypersingular Integral Equations of the First Kind on the Rectangle
}

\author{
Zainidin Eshkuvatov ${ }^{1, *}$, Massamdi Kommuji ${ }^{1}$, Rakhmatullo Aloev $^{2}$, \\ Nik Mohd Asri Nik Long ${ }^{3}$, Mirzoali Khudoyberganov ${ }^{2}$ \\ ${ }^{1}$ Faculty of Science and Technology, Universiti Sains Islam Malaysia, Malaysia \\ ${ }^{2}$ Faculty of Mathematics, National University of Uzbekistan, Uzbekistan \\ ${ }^{3}$ Department of Mathematics, Faculty of Science, Universiti Putra Malaysia, Malaysia
}

Received June 29, 2019; Revised September 17, 2019; Accepted September 23, 2019

Copyright (C) 2020 by authors, all rights reserved. Authors agree that this article remains permanently open access under the terms of the Creative Commons Attribution License 4.0 International License

\begin{abstract}
A hypersingular integral equations (HSIEs) of the first kind on the interval $[-1,1]$ with the assumption that kernel of the hypersingular integral is constant on the diagonal of the domain is considered. Truncated series of Chebyshev polynomials of the third and fourth kinds are used to find semi bounded (unbounded on the left and bounded on the right and vice versa) solutions of HSIEs of first kind. Exact calculations of singular and hypersingular integrals with respect to Chebyshev polynomials of third and forth kind with corresponding weights allows us to obtain high accurate approximate solution. Gauss-Chebyshev quadrature formula is extended for regular kernel integrals. Three examples are provided to verify the validity and accuracy of the proposed method. Numerical examples reveal that approximate solutions are exact if solution of HSIEs is of the polynomial forms with corresponding weights.
\end{abstract}

Keywords Approximation, Chebyshev polynomials, Convergence, Hypersingular integral equations.

\section{Introduction}

Hypersingular integral equations (HSIEs) of the first kind of the form

$$
\frac{1}{\pi} f_{-1}^{1} \varphi(t)\left[\frac{K(x, t)}{(t-x)^{2}}+L_{1}(x, t)\right] d t=f(x), \quad-1<x<1,
$$

encounters in several physical problems such as aerodynamics, hydrodynamics, and elasticity theory (see [1]-[7]).

In 1985, Golberg [1] consider Eq. (1) with the kernel $K(x, t)=1$ and proposed projection method with the truncated series of Chebyshev polynomials of the second kind together with Galerkin and collacation methods. Uniform convergence and the rate of convergence of projection method are obtained in subspace of Hilbert space for HSIEs (1). In 1992, Martin [2] obtained the analytic solution to the simplest one-dimensional hypersingular integral equation i.e. the case of $K(x, t)=1$ and $L_{1}(x, t)=0$ in Eq. (1). In 2006, Mandal and Bera have proposed a simple approximate method (Polynomial approximation) for solving a general hypersingular integral equation of the first kind (1) with $K(x, x) \neq 0$. The method is mostly concentrated with the bounded solution and illustrated proposed method by considering some simple examples. Mandal and Bhattacharya ([4], 2007) proposed approximate numerical solutions of some classes of singular integral equations including HSIEs (1) with $K(x, t)=1$ by using Bernstein polynomials as basis. The method was explained with illustrative examples. Convergence of the method is referred to book of Golberg and Chan [5]. In 2009-2010, Boykov et al. ([6]-[7]) proposed spline-collocation method and its justification for the solution of one-dimensional hypersingular integral equations, poly-hypersingular integral equations, and multi-dimensional hypersingular integral equations. Proved convergence of the method and illustrative examples demonstrated to show the accuracy and efficiency of the developed method. Gulsu and Uzturk ([8], 2014) have purposed approximation method for hypersingular integro-differential equations in the most general form under the mixed conditions in terms of the second kind 
Chebyshev polynomials. This method transforms mixed hypersingular integro-differential equations and the given conditions into matrix equation which corresponding to a system of linear algebraic equation. The error analysis and convergence for the proposed method is also obtained. The reliability and efficiency of the proposed scheme are demonstrated by some numerical experiments. Novin and Fariborzi Araghi ([9], 2019) have applied modification of the homotopy perturbation method (HPM) to HSIEs (1) with $K(x, t)=1, L(x, t)=0$ and compared with the standard homotopy perturbation method. the proposed method can be conveniently fast to get the exact solutions. The validity and reliability of the proposed scheme are discussed. Different examples are included to prove so that proposed method gave exact solutions for all type of characteristic equations. For singular integral equations many efficient methods are derived and proved convergence of the method as well as showed illustrative examples (see Chakrabarti at al. [10]-[11], Dardery and Allan [12], Capobianco et al. [13], Elliot [14]-[15] and so on).

In 2011, Abdulkawi et.al. [16], considered the finite part integral equation (1) with $K(x, t)=1$ and used Chebyshev polynomials of 1st and 2nd kind to find bounded solution of Eq. (1). Exactness of the proposed method for the linear density function are showed and illustrated it with examples. Nik Long and Eshkuvatov [17] have used the complex variable function method to formulate the multiple curved crack problems into hypersingular integral equations of the first kind in more general case and these hypersingular integral equations are solved numerically for the unknown function, which are later used to find the stress intensity factor (SIF). In 2016, Eshkuvatov et al. [18], have used modifed homotopy perturbation method (HPM) to solve Eq. (1) on the interval [- 1, 1] with the assumption that the kernel $K(x, t)$ of the hypersingular integral is constant on the diagonal of the domain. Theoretical and practical examples revealed that the modified HPM dominates the standard HPM, reproducing kernel method and Chebyshev expansion method. Finally, it is found that the modified HPM is exact, if the solution of the problem is a product of weights and polynomial functions. For rational solution the absolute error decreases very fast by increasing the number of collocation points. Eshkuvatov and Narzullaev ([19], accepted in 2018) have solved Eq. (1) using projection method together with Chebyshev polynomials of the first and second kinds to find bounded and unbounded solutions of HSIEs (1) respectively. Existence of inverse of hypersingular operator and exact calculations of hypersingular integral for Chebyshev polynomials allows us to obtain high accurate approximate solution for the case of bounded and unbounded solutions, where the kernel $K(x, t)$ is a constant on the diagonal of the domain $D=[-1,1] \times[-1,1]$.

In this note, HSIEs (1) is considered for the cases of semi-bounded solutions and outlined the collocation method together with Gauss-Chebyshev quadrature formula for regular kernel expansions. The structure of the paper is arranged as follows: In section 2, all the necessary tools are outlined and in Section 3, the details of the derivation of the projection method is presented. Section 4, discusses the existence and uniqueness of the solution in Hilbert space. Finally in Section 4, examples are provided to verify the validity and accuracy of the proposed method, followed by the conclusion in Section 5 .

\section{Preliminaries}

In 2018, Ahdiaghdam ([20]) has solved by using four kind of Chebyshev polynomials for all four cases of solutions (bounded, unbounded, left and right bounded) of singular integral equations (SIEs) of the form

$$
f_{-1}^{1} \frac{\psi(t)}{(t-x)^{\alpha}}+\int_{-1}^{1} K(x, t) \psi(t) d t=f(x), \quad-1<x<1, \quad \alpha \in N
$$

where $K(t, x)$ and $f(x)$ are given real valued Holder continuous functions and $\psi(t)$ is the unknown function to be determined. Convergence of the proposed method is obtained for $\alpha=\{1,2\}$. On the other hand Ahdiaghdam [20] summarized the work in Mason and Handscomb [21] as follows. Let $P_{r, j}(t)$ be the Chebyshev polynomials of the first-forth kind given by

$$
P_{r, j}(t)=\left\{\begin{array}{l}
T_{j}(t)=\cos (j \theta), r=1 \\
U_{j}(t)=\sin ((j+1) \theta) / \sin (\theta), r=2 \\
V_{j}(t)=\cos \left(\left(j+\frac{1}{2}\right) \theta\right) / \cos \left(\frac{\theta}{2}\right), r=3, \\
W_{j}(t)=\sin \left(\left(j+\frac{1}{2}\right) \theta\right) / \sin \left(\frac{\theta}{2}\right), r=4,
\end{array}\right.
$$

where $t=\cos \theta$. The function $P_{r, j}(t)$ satisfies the following orthogonality properties

$$
\mu_{i, j}^{r}=\frac{1}{\pi}\left\langle P_{r, i}, P_{r, j}\right\rangle_{r}=\left\{\begin{array}{l}
0 i \neq j \\
1, i=j=0, r=1 \\
1 / 2, i=j \neq 0, r=1 \\
1 / 2, i=j, r=2 \\
1, i=j, r=\in\{3,4\}
\end{array}\right.
$$


with respect to inner product

$$
\langle f, g\rangle_{r}=\int_{-1}^{1} w_{r}(t) f(t) g(t) d t
$$

where $w_{r}(t), r \in\{1,2,3,4\}$, the weight function defined by

$$
w_{r}(t)=\frac{\lambda_{r}(t)}{\sqrt{1-t^{2}}}, \quad \lambda_{r}(t)=\left\{\begin{array}{l}
1, r=1, \\
1-t^{2}, r=2, \\
1+t, r=3 \\
1-t, r=4
\end{array}\right.
$$

In Mason and Handscomb [21] the following theorem have been proven.

Theorem 1. As a Cauchy principle value integral, we have

$$
S_{r, j}(x)=\frac{1}{\pi} f_{-1}^{1} \frac{w_{r} P_{r, j}(t)}{t-x} d t=\left\{\begin{array}{l}
U_{j-1}(x), r=1 \\
-T_{j+1}(x) r=2 \\
W_{j}(x) r=3 \\
V_{j}(x) r=4
\end{array}\right.
$$

Ahdiaghdam [20] has proved the following statement.

Theorem 2. For $m \geq 1$ derivative of Chebyshev polynomials has the form

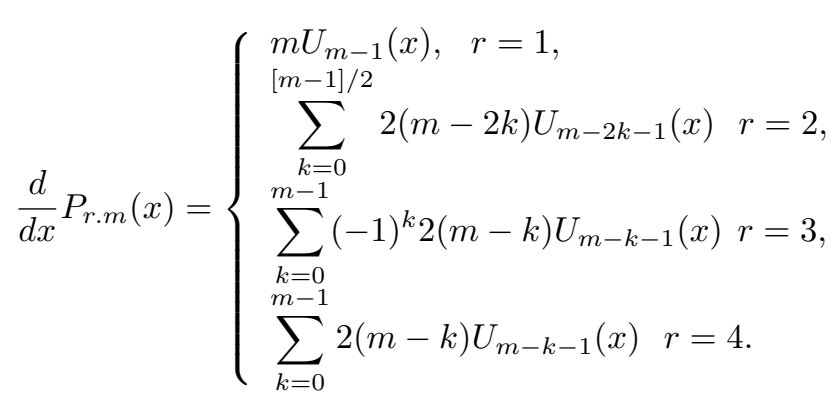

Darbery and Allan [12] summarized three term relations of four kind of Chebyshev polynomials which is given in Mason and Handscomb [21].

$$
\begin{aligned}
& \left\{\begin{array}{l}
\left.T_{0}(x)=1, T_{(} x\right)=x, \\
T_{n}(x)=2 x T_{n-1}-T_{n-2}(x), n \geq 2,
\end{array}\right. \\
& \left\{\begin{array}{l}
\left.U_{0}(x)=1, U_{(} x\right)=2 x, \\
U_{n}(x)=2 x U_{n-1}-U_{n-2}(x), n \geq 2,
\end{array}\right. \\
& \left\{\begin{array}{l}
V_{0}(x)=1, V_{1}(x)=2 x-1, \\
V_{n}(x)=2 x V_{n-1}(x)-V_{n-2}(x), n \geq 2,
\end{array}\right. \\
& \left\{\begin{array}{l}
\left.W_{0}(x)=1, W_{(} x\right)=2 x+1, \\
W_{n}(x)=2 x W_{n-1}-W_{n-2}(x), n \geq 2 .
\end{array}\right.
\end{aligned}
$$

\section{Description of the method}

Since kernel in Eq. (1) is constant on the diagonal we can express it as follows

$$
K(x, t)=c_{0}+(t-x) Q(x)+(t-x)^{2} Q_{1}(x, t), \quad c_{0} \neq 0 .
$$

where $Q(x)$ is smooth function and $Q_{1}(x, t)$ is square integrable kernel. Taking into account Eq. (12) we are able to write Eq. (1) in the form

$$
\frac{c_{0}}{\pi} f_{-1}^{1} \frac{\varphi(t)}{(t-x)^{2}} d t+\frac{Q(x)}{\pi} f_{-1}^{1} \frac{\varphi(t)}{t-x} d t+\frac{1}{\pi} \int_{-1}^{1} L(x, t) \varphi(t) d t=f(x), \quad-1<x<1,
$$


where $L(x, t)=Q_{1}(x, t)+L_{1}(x, t)$, and the first integral in Eq. (13) is being understood as the Hadamard finite-part. Main aim is to find semi-bounded solution of Eq. (13). Hence, we search solution in the form

$$
\varphi(x)=w_{r}(x) u(x), \quad r \in\{3,4\},
$$

where $w_{3}(x)$ and $w_{4}(x)$ are defined by (5). Substituting (14) into (13) yields

$$
\begin{aligned}
\frac{c_{0}}{\pi} \int_{-1}^{1} \frac{w_{r}(t)}{(t-x)^{2}} u(t) d t & +\frac{Q(x)}{\pi} \int_{-1}^{1} \frac{w_{r}(t)}{t-x} u(t) d t \\
& +\frac{1}{\pi} \int_{-1}^{1} w_{r}(x) L(x, t) u(t) d t=f(x), \quad r \in\{3,4\} \quad-1<x<1,
\end{aligned}
$$

Introducing notations

$$
\begin{aligned}
& H_{r} u=\frac{c_{0}}{\pi} f_{-1}^{1} \frac{w_{r}(x)}{(t-x)^{2}} u(t) d t, r \in\{3,4\} \\
& C_{r} u=\frac{Q(x)}{\pi} f_{-1}^{1} \frac{w_{r}(x)}{t-x} u(t) d t, r \in\{3,4\} \\
& L_{r} u=\frac{1}{\pi} \int_{-1}^{1} L(x, t) w_{r}(x) u(t) d t, r \in\{3,4\} .
\end{aligned}
$$

leads to the operator equation

$$
H_{r} u+C_{r} u+L_{r} u=f, \quad r \in\{3,4\}, \quad f \in L_{2 \rho}, u \in L_{1 \rho},
$$

where the spaces $L_{2 \rho}$ and $L_{1 \rho}$ are defined in Section 3.

It is known that the hypersingular operator $H_{g}$ can be considered as differential Cauchy operator i.e.,

$$
H_{g} u=\frac{d}{d x} C_{g} u=\frac{d}{d x}\left(\frac{1}{\pi} f_{-1}^{1} \frac{\omega(t)}{t-x} u(t) d t\right) .
$$

Therefore, Eq. (17) may be viewed as an integro-differential Prandtl's type equation (see [13]). On the other hand from (6) it follows that

$$
\begin{aligned}
& C_{g} V_{m}(x)=\frac{1}{\pi} f_{-1}^{1} \sqrt{\frac{1+t}{1-t}} \frac{V_{m}(t) d t}{(t-x)}=W_{m}(x), m=0,2, \ldots, \\
& C_{g} W_{m}(x)=\frac{1}{\pi} f_{-1}^{1} \sqrt{\frac{1-t}{1+t}} \frac{W_{m}(t) d t}{t-x}=-V_{m}(x), m=0,2, \ldots,
\end{aligned}
$$

In Ahdiaghdam [20] it is shown that differentiating Eq. (19) leads to

$$
\begin{aligned}
& H_{g} V_{m}(x)=\frac{d}{d x} C_{g} V_{m}(x)=\frac{d}{d x} W_{m}(x)=\sum_{k=0}^{m-1} 2(m-k) U_{m-k-1}(x), m=1,2, \ldots, \\
& H_{g} W_{m}(x)=\frac{d}{d x} C_{g} W_{m}(x)=-\frac{d}{d x} V_{m}(x)=\sum_{k=0}^{m-1}(-1)^{k+1} 2(m-k) U_{m-k-1}(x), \quad m=1,2, \ldots,
\end{aligned}
$$

for $m=0$

$$
H_{g} V_{0}(x)=H_{g} W_{0}(x)=0,
$$

Moreover,

$$
\begin{aligned}
& T_{m+1}(x)=\frac{1}{2}\left[U_{m+1}(x)-U_{m-1}(x)\right], m=0,1,2, \ldots, \\
& x U_{m}(x)=\frac{1}{2}\left[U_{m+1}(x)+U_{m-1}(x)\right], m=0,1.2, \ldots \\
& V_{m}(x)=U_{m}(x)-U_{m-1}(x), m=0,1,2, \cdots \\
& \left.W_{m}(x)=(-1)^{m} V_{m}(-x)=U_{m}(x)+U_{m-1}(x)\right), m=0,1,2, \cdots .
\end{aligned}
$$

where $U_{-1}(x)=0$ and $U_{n}(-x)=(-1)^{n} U_{n}(x)$. 
It can easily be shown from (16), (19), (20) and (22) that

$$
\begin{aligned}
& H_{3} V_{m}(x)=c_{0} \sqrt{\frac{\pi}{2}} \sum_{k=0}^{m-1} 2(m-k) \phi_{m-k-1}(x), m=1,2, \cdots \\
& H_{4} W_{m}(x)=-c_{0} \sqrt{\frac{\pi}{2}} \sum_{k=0}^{m-1}(-1)^{k} 2(m-k) \phi_{m-k-1}(x), m=1,2, \cdots \\
& C_{3} V_{m}(x)=Q(x) \sqrt{\frac{\pi}{2}}\left(\phi_{m}(x)+\phi_{m-1}(x)\right), \quad n=0,1, \cdots \\
& C_{4} W_{m}(x)=-\sqrt{\frac{\pi}{2}}\left(\phi_{m}(x)-\phi_{m-1}(x)\right), n=0,1, \cdots
\end{aligned}
$$

where $\phi_{-1}(x)=0$ and

$$
\phi_{m}(x)=\sqrt{\frac{2}{\pi}} U_{m}(x)
$$

Eqs. (23) -(24) are crucial to the rest of our analysis.

To find an approximate solution of Eq.(15), $u(t)$ is approximated by

$$
u(t) \cong u_{n, r}(t)=\sum_{j=0}^{n} b_{j, r} P_{j, r}(t), \quad r \in\{3,4\},
$$

which gives approximate solution of Eq. (13) as follows

$$
\varphi(x) \approx \varphi_{n, r}(x)=\omega_{r}(x) \sum_{j=0}^{n} b_{j, r} P_{j, r}(x), \quad r \in\{3,4\},
$$

where unknown coefficients $b_{j, r}$ need to be defined. To do this end we consider two cases:

- Let $r=3$, i.e. the solution of Eq. (17) is bounded at the left end and unbounded on the right end of the interval $[-1,1]$. Substituting (25) into (15) and using Eqs. (19) - (22) yields

$$
\begin{aligned}
b_{0,3}(Q(x) & \left.+\psi_{0,3}(x)\right) \\
& +\sum_{j=1}^{n} b_{j, 3}\left\{c_{0} \sum_{k=0}^{j-1} 2(j-k) U_{j-k-1}(x)+Q(x)\left(U_{j}(x)+U_{j-1}(x)\right)+\psi_{j, 3}(x)\right\} \\
& =f(x),
\end{aligned}
$$

where $U_{n}(x)$ is the Chebyshev polynomials of the second kind with $U_{-1}(x)=0$ and

$$
\psi_{j, 3}(x)=\frac{1}{\pi} \int_{1}^{1} \sqrt{\frac{1+t}{1-t}} L(x, t) V_{j}(t) d t .
$$

For the collocation method we choose the suitable collocation points $\left\{x_{i}\right\}_{i=1}^{n}$ such as roots of $V_{n+1}(x)$ or $\left(1-x^{2}\right) U_{n-1}(x)$. Then Eq. (27) leads to a system of algebraic linear equations

$$
\begin{aligned}
b_{0,3}\left(Q\left(x_{i}\right)\right. & \left.+\psi_{0,3}\left(x_{i}\right)\right) \\
& +\sum_{j=1}^{n} b_{j, 3}\left\{c_{0} \sum_{k=0}^{j-1} 2(j-k) U_{j-k-1}\left(x_{i}\right)+Q\left(x_{i}\right)\left(U_{j}\left(x_{i}\right)+U_{j-1}\left(x_{i}\right)\right)+\psi_{j, 3}\left(x_{i}\right)\right\} \\
& =f\left(x_{i}\right), i=0,1, \cdots,
\end{aligned}
$$

Solving the system of Eq. (29) for the unknown coefficients $b_{j, 3}, j=0,1, \ldots, n$ and substituting the values of $b_{j, 3}$ into Eq. (26) yields the numerical solution of Eq. (13).

- Let $i=4$, i.e. the solution of Eq. (17) is unbounded at the left end and bounded on the right end of the interval $[-1,1]$. Substitute (25) into (15) for $r=4$ and apply Eqs. (19) - (22) to get

$$
\begin{aligned}
b_{0,4}(Q(x) & \left.+\psi_{0,4}(x)\right) \\
& +\sum_{j=1}^{n} b_{j, 4}\left\{c_{0} \sum_{k=0}^{j-1}(-1)^{k} 2(j-k) U_{j-k-1}(x)-Q(x)\left(U_{j}(x)-U_{j-1}(x)\right)+\psi_{j, 4}(x)\right\}=f(x),
\end{aligned}
$$


where

$$
\psi_{j, 4}(x)=\frac{1}{\pi} \int_{1}^{1} \sqrt{\frac{1-t}{1+t}} L(x, t) W_{j}(t) d t .
$$

To solve Eq. (30) for unknown parameters $b_{j, 2}$ collocation method is used by choosing the suitable node points $\left\{x_{i}\right\}_{i=1}^{n}$ such as roots of $W_{n+1}(x)$ or $\left(1-x^{2}\right) U_{n-1}(x)$. It reduces Eq. (30) to a system of linear equation

$$
\begin{aligned}
b_{0,4}\left(Q\left(x_{i}\right)\right. & \left.+\psi_{0,4}\left(x_{i}\right)\right) \\
& +\sum_{j=1}^{n} b_{j, 4}\left\{c_{0} \sum_{k=0}^{j-1}(-1)^{k} 2(j-k) U_{j-k-1}\left(x_{i}\right)+Q\left(x_{i}\right)\left(U_{j}\left(x_{i}\right)+U_{j-1}\left(x_{i}\right)\right)+\psi_{j, 4}\left(x_{i}\right)\right\} \\
& =f\left(x_{i}\right), i=0,1, \cdots, n,
\end{aligned}
$$

Solving the system of Eq. (32) for the unknown coefficients $b_{j, 4}, j=0,1, \ldots, n$ and substituting the values of $b_{j, 4}$ into Eq. (26) gives the approximate solution of Eq. (13).

\section{Quadrature method}

In the Section 3, we have obtained two types of weighted kernel integrals (28) and (31). It is known that many weighted kernel integrals have not exact solution. So that we need to derive suitable quadrature for numerical computation of weighted kernel integrals.

In this section, we develop Gauss-Chebyshev quadrature rule with Gauss-Lobotto nodes for weighted kernel integrals ((28) and (31)). In Kythe [22], states that the Gauss quadrature formula of the form

$$
\int_{a}^{b} w(x) f(x) d x=\sum_{i=1}^{n+1} A_{i} f\left(x_{i}\right)
$$

is exact for all $f \in P_{2 n+1}$ if the weights $A_{i}$ and the nodes $x_{i}$ can be found from orthogonal polynomials approximation of $f(x)$ and roots of orthogonal polynomials respectively on the interval $[a, b]$.

In particular, if $[a, b]=[-1,1]$ and $w_{3}(t)(x), w_{4}(t)(x)$ are defined by $(6)$ and orthogonal polynomials are the Chebyshev polynomials $V_{n}(t), W_{n}(t)$ of the third and forth kind respectively then resulting formulas of Eq. (33) are known as GaussChebyshev quadrature rule. To derive it let us define the nodes $x_{i, 1}$ and $x_{i, 2}$ as the zeros of $V_{n+1}(x)$ and $W_{n+1}(x)$ respectively,

$$
\begin{gathered}
x_{k, 1}=\cos \left(\frac{(2 k-1) \pi}{2 n+3}\right), \quad k=1,2, \ldots, n+1 \\
x_{k, 2}=\cos \left(\frac{2 k \pi}{2 n+3}\right), \quad i=1,2, \ldots, n+1 .
\end{gathered}
$$

Aghigh et al. [23] states that any Gauss qudrature formula with weight function on $[a, b]$ can be written as follows

$$
\int_{a}^{b} w(x) f(x) d x \approx \sum_{k=1}^{n+1} A_{k} f\left(x_{k}\right), w_{k}=\frac{K_{n+1}}{K_{n}} \frac{\int_{a}^{b} P_{n}^{2}(x) w(x) d x}{P_{n}\left(x_{k}\right) P^{\prime}\left(x_{k-1}\right)},
$$

where $P_{n}(x)$ is a sequence of polynomials orthogonal with respect to weight function $w(x)$ on $[a, b], K_{n}$ denotes the leading coefficients of $P_{n}(x)$ and $\left\{x_{k}\right\}_{k=1}^{n+1}$ are the polynomial roots $\left(P_{n+1}(x)\right)$.

Using (36) and taking into account (34)-(35), Gauss-Chebyshev quadrature rule can be constructed as follows

Lemma 3. (Aghigh et al. [23]) Open Gauss-Chebyshev quadrature rule is given as

$$
\int_{-1}^{1} \sqrt{\frac{1+x}{1-x}} f(x) d x=\sum_{k=1}^{n+1} A_{k, 1} f\left(x_{k, 1}\right)+R_{n+1,1}(f) .
$$

where $A_{k, 1}=\frac{2 \pi}{2 n+3}\left(1+x_{k, 1}\right)$ and $x_{k, 1}$ is defined by (34). Similarly

$$
\int_{-1}^{1} \sqrt{\frac{1-x}{1+x}} f(x) d x=\sum_{k=1}^{n+1} A_{k, 2} f\left(x_{k, 2}\right)+R_{n+1,2}(f) .
$$

where $A_{k, 2}=\frac{2 \pi}{2 n+3}\left(1-x_{k, 2}\right)$ and $x_{k, 2}$ is defined by (35). 
The word "open" is used for not including endpoints. We usually omit "open" since all Gaussian rules with positive weight function are of the open type.

Theorem 4. (Johnson and Riess 1977). Gaussian QF has precision $2 n+1$ only if the points $x_{i}, i=0,1, \ldots, n$ are the zeros of $\phi_{n+1}(x)$, where $\phi_{n+1}(x)$ are orthogonal polynomials.

Theorem 5. (Israilov [25]) If $f \in C^{2 n+2}[a, b]$, then the error of Gaussian $Q F$ is given by

$$
R_{n}(f)=I_{a}^{b}(f)-I_{n}(f)=\frac{f^{2 n+2}(\xi)}{(2 n+2) !} \int_{a}^{b} w(x) \bar{P}_{n+1}^{2}(x) d x, \quad \xi \in[a, b]
$$

where $\bar{P}_{n+1}(x)$ is the monic polynomials of degree $n+1$ with $n+1$ distinct zeros and $w(x)$ is a weight function.

Due to Theorem 5 and referring to Eshkuvatov et al. [19] the following error estimation can be obtained.

Theorem 6. If $f \in C^{2 n+2}[-1,1]$, then the error of Gauss-Chebyshev $Q F s$ (37) and (38) are given by

$$
R_{n+1,1}(f)=R_{n+1,2}(f)=\frac{\pi}{2^{2 n+1}} \frac{f^{2 n+2}\left(\xi_{1}\right)}{(2 n+2) !}, \quad \xi_{1} \in[-1,1],
$$

Now we extend Gauss-Chebyshev QF (37) - (38) for the weight kernel integrals (28) and (31) which are given in Section 3. In many problems of HSIEs regular kernel $L(x, t)$ will be given as convolution type

$$
L(x, t)=\sum_{i=1}^{m} c_{i}(x) d_{i}(t) .
$$

In the case of convolution type kernel (41), the Gauss-Chebyshev QF for the regular kernel in (28) has the form

$$
\begin{aligned}
\psi_{j, 3}(x) & =\frac{1}{\pi} \int_{1}^{1} \sqrt{\frac{1+t}{1-t}} L(x, t) V_{j}(t) d t=\frac{1}{\pi} \sum_{i=1}^{m} c_{i}(x) \int_{1}^{1} \sqrt{\frac{1+t}{1-t}} d_{i}(t) V_{j}(t) d t \\
& =\frac{1}{\pi} \sum_{i=1}^{m} \sum_{k=1}^{n+1} c_{i}(x) A_{k, 1}\left(d_{i}\left(t_{k, 1}\right) V_{j}\left(t_{k, 1}\right)\right), A_{k, 1}=\frac{2 \pi}{2 n+3}\left(1+t_{k, 1}\right),
\end{aligned}
$$

Similarly

$$
\begin{aligned}
\psi_{j, 4}(x) & =\frac{1}{\pi} \int_{1}^{1} \sqrt{\frac{1-t}{1+t}} L(x, t) W_{j}(t) d t=\frac{1}{\pi} \sum_{i=1}^{m} c_{i}(x) \int_{1}^{1} \sqrt{\frac{1-t}{1+t}} d_{i}(t) W_{j}(t) d t \\
& =\frac{1}{\pi} \sum_{i=1}^{m} \sum_{k=1}^{n+1} c_{i}(x) A_{k, 2}\left(d_{i}\left(t_{k, 2}\right) W_{j}\left(t_{k, 2}\right)\right), A_{k, 2}=\frac{2 \pi}{2 n+3}\left(1-t_{k, 2}\right),
\end{aligned}
$$

where $t_{k, 1}$ and $t_{k, 2}$ are defined by (34) and (35) respectively. For non convolution regular kernel $L(x, t)$ case, we have the following Gauss-Chebyshev QF

$$
\begin{aligned}
\psi_{j, 3}(x) & =\frac{1}{\pi} \int_{1}^{1} \sqrt{\frac{1+t}{1-t}} L(x, t) V_{j}(t) d t \\
& =\sum_{k=1}^{n+1} A_{k, 1} f_{1}\left(x, t_{k, 1}\right), A_{k, 1}=\frac{2}{2 n+3}\left(1+t_{k, 1}\right),
\end{aligned}
$$

in the same way we obtain

$$
\begin{aligned}
\psi_{j, 4}(x) & =\frac{1}{\pi} \int_{1}^{1} \sqrt{\frac{1-t}{1+t}} L(x, t) W_{j}(t) d t \\
& =\sum_{k=1}^{n+1} A_{k, 2} f_{2}\left(x, t_{k, 2}\right), A_{k, 1}=\frac{2}{2 n+3}\left(1+t_{k, 2}\right),
\end{aligned}
$$

where

$$
\begin{aligned}
& f_{1}\left(x, t_{k, 1}\right)=L\left(x, t_{k, 1}\right) V_{j}\left(t_{k, 1}\right), \\
& f_{2}\left(x, t_{k, 2}\right)=L\left(x, t_{k, 2}\right) W_{j}\left(t_{k, 2}\right) .
\end{aligned}
$$




\section{Existence of the solution for the semi-bounded cases}

First of all we introduce two type of spaces. First one is weighted Hilbert space. Let $L_{2 \rho_{r}}(-1,1), r \in\{3,4\}$ denote the space of real-valued square integrable functions with respect to weighted functions

$$
\rho_{3}(x)=\sqrt{\frac{1+x}{1-x}}, \rho_{4}(x)=\sqrt{\frac{1-x}{1+x}} .
$$

The spaces $L_{2 \rho_{r}}, r \in\{3,4\}$ are endowed with an inner product

$$
\langle u, v\rangle_{r}=\int_{-1}^{1} \rho_{r}(t) u(t) v(t) d t, \quad r \in\{3,4\},
$$

where the norm $\|u\|_{r}={\sqrt{\langle u, u\rangle_{r}}}, \quad r \in\{3,4\}$.

It is known that $\phi_{n}, n=0,1, \ldots$, defined by (24) are orthonormal polynomials with

$$
\left\|\phi_{n}\right\|_{2 \rho}^{2}=\int_{-1}^{1} \rho(t) \phi_{n}^{2}(t) d t=1, \rho(t)=\sqrt{1-t^{2}},
$$

and the system $\left\{\phi_{k}\right\}_{k=0}^{\infty}$ is a complete orthonormal basis for $L_{2 \rho}$, so that if $u \in L_{2 \rho}$ then

$$
u=\sum_{k=0}^{\infty}\left\langle u, \phi_{k}\right\rangle \phi_{k},
$$

where the sum converges in $L_{2 \rho}$. Consequently, Parseval's equality holds, i.e.

$$
\|u\|_{2 \rho}^{2}=\sum_{k=0}^{\infty}\left\langle u, \phi_{k}\right\rangle^{2} .
$$

Second space for the case of $r=3$ is a subspace of the Hilbert space which is denoted by $L_{1 \rho} \subseteq L_{2 \rho_{3}}$. It consists of all $u \in L_{1 \rho}$ such that

$$
\|u\|_{1 \rho}^{2}=\sum_{m=1}^{\infty} \sum_{k=0}^{m-1} 2^{2}(m-k)^{2}\left\langle u, V_{m}\right\rangle^{2}<\infty .
$$

This subspace can be made themselves into Hilbert space if we define it's inner product of $u \in L_{1 \rho}$ and $v \in L_{1 \rho}$ by

$$
\langle u, v\rangle_{1 \rho}=\sum_{m=1}^{\infty} \sum_{k=0}^{m-1} 2(m-k)\left\langle u, V_{m}\right\rangle\left\langle v, V_{m}\right\rangle .
$$

and hence if $u \in L_{1 \rho}$ then

$$
u=\sum_{m=1}^{\infty}\left\langle u, V_{m}\right\rangle v_{m, k}, \quad v_{m, k}=\sum_{k=0}^{m-1} 2(m-k) U_{m-k-1} .
$$

with the norm

$$
\begin{aligned}
\|u\|_{1 \rho}^{2} & =\langle u, u\rangle=\left\langle\sum_{m=0}^{\infty}\left\langle u, V_{m}\right\rangle v_{m, k}, \sum_{n=0}^{\infty}\left\langle u, V_{n}\right\rangle v_{n, j}\right\rangle \\
& =\left\langle\sum _ { m = 0 } ^ { \infty } 2 ( m + 1 ) \left(\sum_{k=m+1}^{\infty}\left\langle u, V_{k}\right) U_{m}, \sum_{n=0}^{\infty} 2(n+1)\left(\sum_{j=n+1}^{\infty}\left\langle u, V_{j}\right) U_{n}\right\rangle\right.\right. \\
& =\sum_{m=1}^{\infty}\left(\sum_{k=0}^{m-1}[2(m-k)]^{2}\right)\left\langle u, V_{m}\right\rangle^{2} .
\end{aligned}
$$

We do proof that operators $\left(H_{r}+C_{r}+L_{r}\right), r \in\{3,4\}$ in Eq. (17) are invertible. To do these end we consider each case separately. 
- For the case $r=3$, we extend the operator $H_{3}$ defined by (16) as a bounded operator from $L_{1 \rho}$ to $L_{2 \rho_{3}}$. Let

$$
u=\sum_{m=0}^{\infty}\left\langle u, V_{m}\right\rangle V_{m}
$$

then using (20) we obtain

$$
H_{3} u=\sum_{m=0}^{\infty}\left\langle u, V_{m}\right\rangle H_{3} V_{m}=c_{0} \sum_{m=1}^{\infty}\left(\sum_{k=0}^{m-1} 2(m-k) U_{m-k-1}\right)\left\langle u, V_{m}\right\rangle .
$$

Restructuring the infinite sum in Eq. (54) and use orthogonality condition of Chebyshev polynomials of the 2nd kind we arrive at

$$
H_{3} u=c_{0} \sum_{m=0}^{\infty} 2(m+1)\left(\sum_{j=m+1}^{\infty}\left\langle u, V_{j}\right\rangle\right) U_{m}
$$

and observe that

$$
\begin{aligned}
\left\|H_{3} u\right\|_{2 \rho_{3}}^{2} & =c_{0}^{2} \sum_{m=0}^{\infty}[2(m+1)]^{2}\left(\sum_{j=m+1}^{\infty}\left\langle u, V_{j}\right\rangle\right)^{2} \\
& =c_{0}^{2} \sum_{m=1}^{\infty} \sum_{k=0}^{m-1} 2^{2}(m-k)^{2}\left\langle u, V_{m}\right\rangle^{2}=c_{0}^{2}\|u\|_{1 \rho}^{2} .
\end{aligned}
$$

From this it follows that

$$
\|H u\|_{2 \rho_{3}}=\left|c_{0}\right|\|u\|_{1 \rho} .
$$

So that $H_{3}$ is an isometry with constant multiplicity. It is not hard to show that $H_{3}^{-1}: L_{1 \rho} \rightarrow L_{2 \rho_{3}}$ exists and is given by

$$
H_{3}^{-1} u=\frac{1}{c_{0}} \sum_{m=0}^{\infty}\left(\frac{\left\langle u, V_{m}\right\rangle}{-(m+1)}\right)\left(V_{m}-\frac{1}{m} U_{m-1}\right)
$$

hence $H_{3}$ is invertible.

Lemma 7. The norm of operator $H_{3}^{-1}: L_{1 \rho} \rightarrow L_{2 \rho_{3}}$ is

$$
\left\|H_{3}^{-1}\right\|=\frac{1}{\left|c_{0}\right|}
$$

Lemma 7 can be proven in a similar way as given in Eshkuvatov et al. [18].

Lemma 8. Let $A, B$ be operators acting in Hilbert space. If $A$ is bounded and $B$ is compact then the products $A B$ and $B A$ are compact.

Lemma 2 is proven in Reed and Simon [26, Theorem VI.12, pp. 200].

Lemma 9. The operators $C_{3}: L_{1, \rho} \rightarrow L_{2 \rho_{3}}$ and $H_{3}^{-1} C_{3}: L_{1 \rho} \rightarrow L_{2 \rho_{3}}$ are compact.

It can be proved by following Eshkuvatov et al. [18].

Assumption 10. $\lambda=1$ does not belong to the null space of $N\left(I+\lambda H^{-1}(C+L)\right)=\{0\}$.

Lemma 11. Let the Assumption 4 is satisfied, then the operator $H_{3}+C_{3}+L_{3}$ is invertible, and the main equation (17) for the case $r=3$ has a unique solution.

Proof of the Lemma 11: Consider more general HSIEs of the form.

$$
\left[H_{3}+\lambda\left(C_{3}+L_{3}\right)\right] u=f
$$

For $\lambda=1$ it returns to Eq. (17). Let us rewrite Eq. (59) in the form

$$
\left[I+\lambda H_{3}^{-1}\left(C_{3}+L_{3}\right)\right] u=H_{3}^{-1}(f) .
$$


Due to (57) operator $H_{3}$ is invertible therefore it is bounded. If we are able to show that operator $I+\lambda H_{3}^{-1}\left(C_{3}+L_{3}\right)$ are invertible then operator equation Eq. (60) has a unique solution.

Let us define operators $T_{r}, T_{l}: L_{1, \rho} \rightarrow L_{2 \rho_{3}}$ as

$$
\begin{aligned}
& T_{1} u=\sum_{m=0}^{\infty}\left\langle u, \phi_{m}\right\rangle \phi_{m}, \quad T_{2} u=\sum_{m=1}^{\infty}\left\langle u, \phi_{m}\right\rangle \phi_{m-1}, \\
& T_{3} u=\sum_{m=1}^{\infty}\left\langle u, \phi_{m-1}\right\rangle \phi_{m}, \quad T_{4} u=\sum_{m=1}^{\infty}\left\langle u, \phi_{m-1}\right\rangle \phi_{m-1} .
\end{aligned}
$$

From Eq. (23) it can be easily shown that

$$
C_{3} u=C_{3}\left(\sum_{m=0}^{\infty}\left\langle u, V_{m}\right\rangle V_{m}\right)=\sqrt{\frac{\pi}{2}} Q(x)\left(T_{1}+T_{2}+T_{3}+T_{4}\right) u
$$

These operators $T_{r}, r \in\{1,2,3,4\}$ are bounded from $L_{1, \rho} \rightarrow L_{2 \rho_{3}}$. Moreover, boundedness and the compactly embeddability of $T_{r}$ from $L_{1, \rho} \rightarrow L_{2 \rho_{3}}$ (Berthold Berthold et al. (1992, Conclusion 2.3)) implies the compactness of operators $T_{r}, r \in\{1,2,3,4\}$.

Since operators $T_{r}$ are compact, its linear combinations is also compact. As we know $Q(x)$ is a continues function on the closed interval $[-1,1]$ and $T_{r}$ are compact, their product $C_{3}$ is also compact due to Lemma 8 . Since operators $C_{3}$ and $L_{3}$ are compact then $C_{3}+L_{3}: L_{1}(\rho) \rightarrow L_{1}(\rho)$ is also compact. We know that $H_{3}^{-1}\left(C_{3}+L_{3}\right): L_{1}(\rho) \rightarrow L_{1}(\rho)$ is a compact operator due to Lemma 8.

Due to the Fredholm theorem Reed and Simon (1980, Teorem VI.14) the inverse operator $\left.\left(I+\lambda H_{3}^{-1}\left(C_{3}+L_{3}\right)\right)^{[-1}\right]$ of the operator function $\left(I+\lambda H_{3}^{-1}\left(C_{3}+L_{3}\right)\right)$, exists for all $\lambda \in C C_{1}$, where $C_{1}$ is a discrete subset of $C$ (i.e. a set $C_{1}$ has no limit points in $C)$ and for $\lambda \in C_{1}$ the null space $N\left(I+H_{3}^{-1}\left(C_{3}+L_{3}\right)\right)$ is finite, that is $z=\lambda^{-1}$ is the eigenvalue of $H_{3}\left(C_{3}+L_{3}\right)$ with finite multiplicity. These facts allows us to suppose that $\lambda=1$ does not belong to $C_{1}$. Thus we have proved that (15) is solvable and has unique solution for the case $r=3$.

In the case of $r=4$ corresponding changes can be done to provide the existence and uniqueness of the solutions of HSIEs (17).

\section{Numerical Results}

We very often need to use polynomial values of the Chebyshev polynomials for the numerical computation. Table 1 refers to the first few function of Chebyshev polynomials of first $T_{n}(x)$ and second kinds $U_{n}(x)$ respectively.

Table 1: Chebyshev polynomials of the first and second kind

\begin{tabular}{|c|c|c|}
\hline $\mathrm{n}$ & $T_{n}(x)$ & $U_{n}(x)$ \\
\hline 0 & 1 & 1 \\
\hline 1 & $x$ & $2 x$ \\
\hline 2 & $2 x^{2}-1$ & $4 x^{2}-1$ \\
\hline 3 & $4 x^{3}-3 x$ & $8 x^{3}-4 x$ \\
\hline 4 & $8 x^{4}-8 x^{2}+1$ & $16 x^{4}-12 x^{2}+1$ \\
\hline 5 & $16 x^{5}-20 x^{3}+5 x$ & $32 x^{5 k}-32 x^{3}+6 x$ \\
\hline 6 & $32 x^{6}-48 x^{4}+18 x^{2}-1$ & $64 x^{6}-80 x^{4}+24 x^{2}-1$ \\
\hline
\end{tabular}

Table 2 refers to the first few function of Chebyshev polynomials of third $V_{n}(x)$ and forth kinds $W_{n}(x)$.

\subsection{Case $1 r=3$. Bounded solution on the left and unbounded solution on the right}

Example 1: Let us investigate the following HSIEs.

$$
\frac{1}{\pi} \int_{-1}^{1} \frac{(1+2(t-x))}{(t-x)^{2}} \varphi(t) d t+\frac{1}{\pi} \int_{-1}^{1}\left(\frac{1}{2} e^{2 x} t^{3}\right) \varphi(t) d t=f(x),
$$

where

$$
f(x)=-32 x^{3}-32 x^{2}+24 x+4+\frac{1}{2} e^{2 x} .
$$


Table 2: Chebyshev polynomials of the third and forth kind

\begin{tabular}{|l|c|c|}
\hline$n$ & $V_{n}(x)$ & $W_{n}(x)$ \\
\hline 0 & 1 & 1 \\
\hline 1 & $2 x-1$ & $2 x+1$ \\
\hline 2 & $4 x^{2}-2 x-1$ & $4 x^{2}+2 x-1$ \\
\hline 3 & $8 x^{3}-4 x^{2}-4 x+1$ & $8 x^{3}+4 x^{2}-4 x-1$ \\
\hline 4 & $16 x^{4}-8 x^{3}-12 x^{2}+4 x+1$ & $16 x^{4}+8 x^{3}-12 x^{2}-4 x+1$ \\
\hline 5 & $32 x^{5}-16 x^{4}+32 x^{3}+12 x^{2}+6 x-1$ & $32 x^{5}-16 x^{4}-32 x^{3}-12 x^{2}+6 x+1$ \\
\hline 6 & $64 x^{6}-32 x^{5}-80 x^{4}+32 x^{3}+24 x^{2}-6 x-1$ & $64 x^{6}+32 x^{5}-80 x^{4}-32 x^{3}+24 x^{2}+6 x-1$ \\
\hline
\end{tabular}

The exact solution of Eq. (62) is

$$
\varphi(x)=\sqrt{\frac{1+x}{1-x}}\left(-16 x^{3}+24 x^{2}-12 x+8\right) .
$$

Solution: Comparing (62) with (13) we get

$$
c_{0}=1, \quad Q(x)=2, \quad L(x, t)=\frac{1}{2} e^{2 x} t^{3} .
$$

From (27)-(28) and (64) it follows that

$$
\begin{aligned}
b_{0,3}(2+ & \left.\psi_{0,3}(x)\right) \\
& +\sum_{j=1}^{n} b_{j, 3}\left\{\sum_{k=0}^{j-1}(-1)^{k} 2(j-k) U_{j-k-1}(x)+2\left(U_{j}(x)+U_{j-1}(x)\right)+\psi_{j, 3}(x)\right\} \\
& =-32 x^{3}-32 x^{2}+24 x+4+\frac{1}{2} e^{2 x} .
\end{aligned}
$$

where $U_{-1}(x)=0$ and

$$
\psi_{j, 3}(x)=\frac{1}{\pi} \int_{1}^{1}\left(\frac{1}{2} e^{2 x} t^{3}\right) \sqrt{\frac{1+t}{1-t}} V_{j}(t) d t
$$

It can be easily obtain that

$$
t^{3}=\frac{3}{8} V_{0}(t)+\frac{3}{8} V_{1}(t)+\frac{1}{8} V_{2}(t)+\frac{1}{8} V_{4}(t) .
$$

Using (66) and orthogonality conditions (4) we obtain

$$
\psi_{0,3}(x)=\frac{3}{16} e^{2 x}, \psi_{1,3}(x)=\frac{3}{16} e^{2 x}, \psi_{2,3}(x)=\frac{1}{16} e^{2 x}, \psi_{3,3}(x)=\frac{1}{16} e^{2 x}, \psi_{j, 3}(x)=0, j \geq 4 .
$$

Substituting (68) into (65) and equating like powers of $x$ leads to

$$
b_{0,3}=8, b_{1,3}=-6, b_{2,3}=4, b_{3,3}=-2
$$

which leads to identical exact solution

$$
\begin{aligned}
\varphi(x) & =\frac{1+x}{1-x}\left[b_{0,3} V_{0}(x)+b_{1,3} V_{1}(x)+b_{2,3} V_{2}(x)+b_{3,3} V_{3}(x)\right] \\
& =\sqrt{\frac{1+x}{1-x}}\left(-16 x^{3}+24 x^{2}-12 x+8\right)
\end{aligned}
$$

To find approximate solution substitute (68) into (65) and choose collocation points $x_{i}$ as the root of $V_{n+1}(x)$ which are

$$
x_{i}=\cos \frac{(2 i+1) \pi}{2 n+3}, i=0, \ldots, n
$$


it follows that the system of algebraic equations (65) has the form

$$
\begin{aligned}
b_{0,3}(2+ & \left.\frac{3}{16} e^{2 x_{i}}\right) \\
& +\sum_{j=1}^{n} b_{j, 3}\left\{\sum_{k=0}^{j-1}(-1)^{k} 2(j-k) U_{j-k-1}\left(x_{i}\right)+2\left(U_{j}\left(x_{i}\right)+U_{j-1}\left(x_{i}\right)\right)+\psi_{j, 3}\left(x_{i}\right)\right\} \\
& =-32 x_{i}^{3}-32 x_{i}^{2}+24 x_{i}+4+\frac{1}{2} e^{2 x_{i}}, i=0,1, \cdots n .
\end{aligned}
$$

Solving Eq. (72) at the collocation points (71) for the different value of $n$, we obtain the numerical solution of Eq. (62). The errors of numerical solution of Eq. (62) are summarized in Table 3. The results in Table 3 shows that method proposed is exact

Table 3: Numerical solution of Example 1

\begin{tabular}{|c|c|c|c|c|}
\hline$x$ & Errors $R_{n}$, with $n=5$ & Errors $R_{n}$, for $n=6$ & Errors $R_{n}$, for $n=8$ & Errors $R_{n}$, for $n=10$ \\
\hline-0.9999 & 0 & 0 & $7.7842 \times 10^{-19}$ & $1.1469 \times 10^{-19}$ \\
-0.901 & 0 & 0 & $2.1755 \times 10^{-17}$ & $3.2053 \times 10^{-18}$ \\
-0.725 & 0 & 0 & $3.2162 \times 10^{-17}$ & $4.7386 \times 10^{-18}$ \\
-0.436 & 0 & 0 & $2.9765 \times 10^{-17}$ & $4.3855 \times 10^{-18}$ \\
-0.015 & 0 & 0 & $3.1517 \times 10^{-17}$ & $4.6436 \times 10^{-18}$ \\
0.015 & 0 & 0 & $3.1502 \times 10^{-17}$ & $4.6414 \times 10^{-18}$ \\
0.436 & 0 & 0 & $3.3535 \times 10^{-17}$ & $4.9410 \times 10^{-18}$ \\
0.725 & 0 & 0 & $4.1357 \times 10^{-17}$ & $6.0937 \times 10^{-18}$ \\
0.901 & 0 & 0 & $6.2334 \times 10^{-17}$ & $9.1842 \times 10^{-18}$ \\
0.9999 & 0 & 0 & $1.8389 \times 10^{-15}$ & $2.7096 \times 10^{-16}$ \\
\hline
\end{tabular}

however the roots has many level of square roots $($ i.e $: \sqrt{23+\sqrt{2}})$ it makes the equation more complicated and leads to a system of equations that cannot solve for large $n$. However, the numerical results are decreased as $n$ increased. Table 3 reveals that method proposed is very accurate and it coincides with exact solution when we choose five terms $n=5$ only.

\subsection{Case 2, $r=4$. Bounded solution on the right and unbounded solution on the left}

Example 2: Consider the following HSIEs of the form

$$
\frac{1}{\pi} \int_{-1}^{1} \frac{\varphi(t)}{(t-x)^{2}} d t+\frac{1}{\pi} \int_{-1}^{1} \frac{x \sin t}{t} \varphi(t) d t=f(x),
$$

where $f(x)=-4 x^{2}+2 x-2$ and exact solution of Eq. (73) is not known.

Solution: Approximate solution is searched as

$$
\varphi(x)=\varphi_{n, 4}(x)=\sqrt{\frac{1-x}{1+x}} \sum_{j=0}^{3} b_{j, 4} W_{j}(x) .
$$

Substitute (74) into (73) with $n=3$ yields

$$
\sum_{j=0}^{3} b_{j, 4}\left\{\frac{1}{\pi} \int_{-1}^{1} \frac{1-t}{1+t} \frac{W_{j}(t)}{(t-x)^{2}} d t+\frac{1}{\pi} \int_{-1}^{1} \frac{1-t}{1+t} \frac{x \sin t}{t} W_{j}(t) d t\right\}=f(x) .
$$

Using Maclaurin series for sint we obtain

$$
\frac{\sin t}{t} \approx 1-\frac{t^{2}}{3 !}+\frac{t^{4}}{5 !}+\frac{t^{6}}{7 !}
$$

Applying (19) - (22) and taking into account Table 2, we get

$$
\begin{gathered}
b_{0,4} \psi_{0,4}(x)+b_{1,4}\left[-2+\psi_{1,4}(x)\right]+b_{2,4}\left[-(8 x-2)+\psi_{2,4}(x)\right] \\
+b_{3,4}\left[-\left(24 x^{2}-8 x-4\right)+\psi_{3,4}(x)\right]=f(x)
\end{gathered}
$$


where

$$
\psi_{j, 4}(x)=\frac{1}{\pi} \int_{-1}^{1} x\left[1-\frac{t^{2}}{3 !}+\frac{t^{4}}{5 !}+\frac{t^{6}}{7 !}\right] \sqrt{\frac{1-t}{1+t}} W_{j}(t) d t
$$

Since

$$
1-\frac{t^{2}}{3 !}+\frac{t^{4}}{5 !}+\frac{t^{6}}{7 !}=c_{0} W_{0}(t)+c_{1} W_{1}(t)+c_{2} W_{2}(t)+c_{3} W_{3}(t)+c_{4} W_{4}(t)+c_{5} W_{5}(t)+c_{6} W_{6}(t)
$$

By equating the same powers of $t$ we find

$$
\begin{aligned}
& c_{0}=0.9197, \quad c_{1}=0.0396, \quad c_{2}=-0.0396, \quad c_{3}=-5.0223 e^{-04} \\
& c_{4}=5.0223 e^{-04}, \quad c_{5}=3.1002 e^{-06}, \quad c_{6}=-3.1002 e^{-06} .
\end{aligned}
$$

Substitutions (79) - (80) into (78) and orthogonality conditions (4) yields

$$
\begin{aligned}
& \psi_{0,4}(x)=0.9197 x, \psi_{1,4}(x)=0.0396 x, \quad \psi_{2,4}(x)=-0.0396 x, \quad \psi_{3,4}(x)=-5.0223 e^{-04} x \\
& \psi_{4,4}(x)=5.0223 e^{-04} x, \psi_{5,4}(x)=3.1002 e^{-06} x, \quad \psi_{6,4}(x)=-3.1002 e^{-06} x, \quad \psi_{j, 4}(x)=0, j \geq 7 .
\end{aligned}
$$

By equating like powers of $x$ in (77) from both sides we find that

$$
b_{0,4}=8.6985 C+0.6676, \quad b_{1,4}=C+\frac{4}{3}, \quad b_{2,4}=C, \quad b_{3,4}=\frac{1}{6}, \quad b_{j, 4}=0, j \geq 4 .
$$

Exact solution is achieved when we substitute Eq. (82) into (74) i.e.

$$
\varphi(x)=\sqrt{\frac{1-x}{1+x}}\left(8.6985 C+0.6676+\left(C+\frac{4}{3}\right) W_{1}(x)+C W_{2}(x)+\frac{1}{6} W_{3}(x)\right) .
$$

The roots of $W_{n+1}(x)=0$ leads

$$
x_{k}=\cos \left(\frac{2 k}{2 n+3} \pi\right), k=1,2, \ldots, n+1
$$

Solving (77) at collocation points (84) yields

$$
b_{0}=0.6675, b_{1}=\frac{4}{3}, b_{2}=0, b_{3}=\frac{1}{6}
$$

The error of numerical solution of (73) are summarised in Table 4.

Table 4. Error term of Example 2

\begin{tabular}{|c|c|c|c|}
\hline$x$ & Exact & Approximate & Error \\
\hline-0.9999 & -117.6502 & -117.6530 & $2.8284 \times 10^{-3}$ \\
-0.901 & -1.7606 & -1.7607 & $8.7640 \times 10^{-5}$ \\
-0.725 & 0.5675 & 0.5674 & $5.0091 \times 10^{-5}$ \\
-0.436 & 1.5613 & 1.5613 & $3.1913 \times 10^{-5}$ \\
-0.015 & 1.8317 & 1.8317 & $2.0302 \times 10^{-5}$ \\
0.015 & 1.8367 & 1.8366 & $1.9702 \times 10^{-5}$ \\
0.436 & 1.8447 & 1.8447 & $1.2534 \times 10^{-5}$ \\
0.725 & 1.6541 & 1.6541 & $7.9855 \times 10^{-6}$ \\
0.901 & 1.1759 & 1.1759 & $4.5641 \times 10^{-6}$ \\
0.9999 & 0.0413 & 0.0413 & $1.4142 \times 10^{-7}$ \\
\hline
\end{tabular}

Example 3: Let us consider the following HSIEs.

$$
\frac{1}{\pi} \int_{-1}^{1} \frac{\varphi(t)}{(t-x)^{2}} d t+\frac{1}{\pi} \int_{-1}^{1}\left(t e^{x}\right) \varphi(t) d t=-4 x+\frac{1}{4} e^{x},
$$

The exact solution of Eq. (86) given by Ahdiaghdam (2018) is

$$
\varphi(x)=\sqrt{1-x^{2}}(2 x) .
$$


which is in the form of Chebyshev polynomials of second kind. In this work, we obtain the solution in the form of Chebyshev polynomials of fourth kind.

Solution: The approximate solution is searched by choosing $\mathrm{n}=5$ as follows

$$
\varphi(x)=\varphi_{n, 4}(x)=\sqrt{\frac{1-x}{1+x}} \sum_{j=0}^{5} b_{j, 4} W_{j}(x) .
$$

Subtitute Eq. (88) into Eq. (86) and from Eq. (30) - Eq. (31) it follows that

$$
b_{0,4}\left(\psi_{0,4}(x)\right)+\sum_{j=1}^{5} b_{j, 4}\left\{\sum_{k=0}^{j-1}(-1)^{k} 2(j-k) U_{j-k-1}(x)+\psi_{j, 4}(x)\right\}=-4 x+\frac{1}{4} e^{x},
$$

where

$$
\psi_{j, 4}(x)=\frac{1}{\pi} \int_{1}^{1} t e^{x} \sqrt{\frac{1-t}{1+t}} W_{j}(t) d t .
$$

Collocation method is used to solve Eq. (89) for unknown parameters $b_{j, 2}$. Collocation points $\left\{x_{i}\right\}_{i=0}^{5}$ are chosen as roots of $W_{n+1}(x)$ or $\left(1-x^{2}\right) U_{n-1}(x)$ and reduces Eq. (89) to a system of linear equation

$$
b_{0,4}\left(\psi_{0,4}\left(x_{i}\right)\right)+\sum_{j=1}^{5} b_{j, 4}\left\{\sum_{k=0}^{j-1}(-1)^{k} 2(j-k) U_{j-k-1}\left(x_{i}\right)+\psi_{j, 4}\left(x_{i}\right)\right\}=-4 x_{i}+\frac{1}{4} e^{x_{i}}
$$

Solving the system of Eq. (91) obtains

$$
b_{0,4}=0, b_{1,4}=\frac{1}{2}, b_{2,4}=\frac{1}{2}, b_{3,4}=0, b_{4,4}=0, b_{5,4}=0 .
$$

Subtitute result in Eq. (88) obtains

$$
\varphi(x)=\varphi_{5,4}(x)=\sqrt{\frac{1-x}{1+x}}\left(2 x^{2}+2 x\right),
$$

which is the solution for the Eq. (86) in the form of Chebyshev polynomials of fourth kind.

\section{Conclusions}

In this note, we have developed projection method for solving HSIEs of the first kind, where the kernel $K(x, t)$ is constant on the diagonal of the rectangle region $D$. Collocation method are used to obtain a system of algebraic equations for the unknown coefficients. Examples 1 and 2 verify that the developed method is very accurate and stable for HSIEs of the first kind. Numerical solution are obtained with the help of Matlab software.

\section{Acknowledgment}

This work was supported by Universiti Sains Islam Malaysia (USIM) under RMC Research Grant Scheme (FRGS, 2018). Project code is USIM/FRGS/FST/055002/51118.

\section{REFERENCES}

[1] M.A. Golberg (1985), The convergence of several algorithms for solving integral equations with finite-part integrals, II, J. Integral Equations and Its Applications. Vol. 9(3), 1985, pp. 267-275.

[2] P.A. Martin, Exact solution of a simple hypersingular integral equation. J Integral Equ Appl, Vol. 4(2), 1992, pp. 197-204.

[3] B.N.Mandal, G.H. Bera, Approximate solution for a class of hypersingular integral equations. Appl. Math. Lett., Vol. 19, 2006, pp. 1286-1290. 
[4] B.N.Mandal, S. Bhattacharya, Numerical solution of some classes of integral equations using Bernstain polynomials. Appl. Math. Comput, Vol. 190, 2007, pp. 1707-1716.

[5] M.A. Golberg, C.S. Chen, Discrete Projection Methods for Integral Equations, Computational Mechanics Publications, Southampton, 1997.

[6] I.V. Boykov, E.S. Ventsel, A.I. Boykova, An approximate solution of hypersingular integral equations. Applied Numerical Mathematics, Vol. 60(6), 2010, 607- 628.

[7] I.V. Boykov, E.S. Ventsel, A.I. Boykova, Accuracy Optimal Methods for Evaluating Hypersingular Integrals, Appl. Num. Math., Vol. 59, 2009, pp. 1366-1385.

[8] M. Gulsu, Y. Ozturk, Numerical approach for the solution of hypersingular integro-differential equations. Applied Mathematics and Computation 230 (2014), pp. 701-710.

[9] R. Novin and M. A. Fariborzi Araghi. Hypersingular integral equations of the first kind: A modified homotopy perturbation method and its application to vibration and active control. Journal of Low Frequency Noise, Vibration and Active Control. DOI: $10.1177 / 1461348419827378,2019$, pp. 1-22.

[10] A. Chakrabarti, G. Vanden Berghe. "Approximate solution of singular integral equations.” Applied mathematics letters, Vol. 17(5), 2004, pp. 553-559.

[11] A. Chakrabarti, R. Manam, Solution of a Logarithmic Singular Integral Equation. Applied Mathematics Letters. Vol.16, 2003, pp. 369373.

[12] S. M. Dardery, M. M. Allan, Chebyshev Polynomials for Solving a Class of Singular Integral Equations. Applied Mathematics, 2014, 5, 753-764.

[13] M. R. Capobianco, G. Criscuolo, P. Junghanns, U. Luther, Uniform convergence of the collocation method for Prandtls integro-differential equation, ANZIAM J., 42 (2000), 151-168

[14] D. Elliot, The classical collocation method for singular integral equations, SIAM J. Numer. Anal. Vol. 19, 1982, pp. 816-832.

[15] D. Elliot, Rates of convergence for the method of classical collocation for singular integral equations, SIAM J. Numer. Anal. Vol. 21, 1984, pp. 136-148.

[16] M. Abdulkawi, N.M.A. Nik Long, Z. K. Eshkuvatov. Numerical Solution of Hypersingular Integral Equations. Int J. Pure Appl. Math, Vol. 69(3), 2011, pp. 265-274.

[17] N.M.A. Nik Long, Z.K. Eshkuvatov. Hypersingular intergral equations for multiple curved cracks in plane elasticty. International Journal of Solids and Structures. Vol. 46(13), 2009, pp. 2611-2617.

[18] Z. K. Eshkuvatov, F. S. Zulkarnain, N. M. A. Nik Long and Z. Muminov (2016), Modified homotopy perturbation method for solving hypersingular integral equations of the first kind. SpringerPlus, 5:1473, pp. 1-21 (2016). doi: 10.1186/s40064-016-3070-z.

[19] Z.K. Eshkuvatov, Anvar Narzullaev, Projection Method for Bounded and Unbounded Solutions of Hypersingular Integral Equations of the First Kind. Indian Journal of Industrial and Applied Mathematics. To be appeared.

[20] Samad Ahdiaghdam (2018), Solving singular integral equations by using orthogonal polynomials. Computational Methods for Differential Equations. Vol. 6(4), pp. 411-425.

[21] J.C. Mason and D.C. Handscomb (2003). Chebyshev polynomials, CRC Press LLC.

[22] Kythe P.K. Handbook of Computational Methods for Integration. Chapman, Hall/CRC. 2005.

[23] K. Aghigh, M. Masjed-Jamei, M. Dehghan, A survey on third and fourth kind of Chebyshev polynomials and their applications. Applied Mathematics and Computation Vol. 199, 2008, pp. 2-12.

[24] I.K. Lifanov, L.N. Poltavskii, G.M. Vainikko, Hypersingular Integral Equations and Their Applications, Chapman Hall/CRC, CRC Press Company, Boca Raton, London, New York, Washington, DC, 2004.

[25] Israilov M.I. Numerical methods. Vol. 1, Press FAN, 2002. (Uzbek)

[26] M. Reed, B. Simon, Functional Analysis, Vol. 1 (Methods of Modern Mathematical Physics). Academic press, inc. 1980. 